\title{
COMPARING OPTICAL-FLOW BASED METHODS FOR QUANTIFICATION OF MYOCARDIAL DEFORMATIONS ON RT3D ULTRASOUND
}

\author{
Qi Duan ${ }^{1}$, Elsa Angelini ${ }^{2}$, Olivier Gerard ${ }^{3}$, Shunichi Homma ${ }^{4}$, Andrew Laine ${ }^{1}$ \\ ${ }^{1}$ Department of Biomedical Engineering, Columbia University, New York, NY, USA \\ ${ }^{2}$ Ecole Nationale Supérieure des Télécommunications, 46 rue Barrault, Paris, France, 75013 \\ ${ }^{3}$ Philips France, 51 rue Carnot, BP 301, Suresnes, France, 92156 \\ ${ }^{4}$ Department of Medicine, Columbia University, New York, NY, USA
}

\begin{abstract}
Quantitative analysis of cardiac motion is of great clinical interest in assessing ventricular function. Real-time 3-D (RT3D) ultrasound transducers provide valuable threedimensional information, from which quantitative measures of cardiac function can be extracted. Such analysis requires segmentation and visual tracking of the left ventricular endocardial border. Previously, we presented a method based on four-dimensional optical flow motion estimation for temporal tracking of ventricular borders in RT3D ultrasound. A myocardial displacement field and dynamic cardiac metrics were computed by interpolating the boundary tracking results. In this paper, we propose three additional methods for deriving dynamic cardiac information from tracking ventricular surfaces and demonstrate these methods on a clinical dataset.
\end{abstract}

\section{INTRODUCTION}

Ultrasound is the cardiac screening modality with the highest temporal resolution, but is still limited to twodimensions in most hospitals and clinical centers. Development of 3D echocardiography started in the 1990s [1], with real-time 3D (RT3D) ultrasound based on matrix phased arrays. Recently, a new generation of RT3D transducers was introduced by Philips Medical Systems (Best, The Netherlands) with the SONOS 7500 transducer followed by the IE33 model that acquires a fully sampled cardiac volume within four cardiac cycles. This technical design increased the spatial resolution and image quality, which makes 3D ultrasound techniques increasingly attractive for routine cardiac clinical diagnosis cardiac patients. Since RT3D ultrasound acquires volumetric ultrasound sequences with fairly high temporal resolution using a fixed-positioned transducer, it can capture complex 3D cardiac motion well.

Cardiac motion analysis from clinical image sequences has been an active research area over the past decade. However, most research has been based on CT and MRI data. Previous efforts using ultrasound data for motion analysis include intensity-based optical flow (OF) tracking, strain-imaging, and elastography. In [2] for example, a shape tracking approach was used to track endocardial surface patches in $3 \mathrm{D}$ echocardiography for motion analysis.

In a previous study [3, 4], we developed a framework to derive several dynamic cardiac measures based on fourdimensional optical flow using RT3D ultrasound sequences. This framework was applied to a clinical data set from a heart transplant patient and dynamic measurements agreed with clinical evaluation. In that framework, myocardial motion fields were estimated by radial basis function (RBF) interpolation of ventricular surfaces (i.e. endocardium and epicardium). However, we wanted to determine how accurate the estimated myocardial motion field which was derived from the interpolation of the epicardial and endocardial motion fields was in comparison to direct tracking of the myocardial points. To answer that question as well as to improve our dynamical cardiac computational framework, we investigated three alternative computational schemes for deriving myocardial motion fields and dynamic cardiac metrics based on $\mathrm{OF}$.

\section{METHODOLOGY}

To suppress the speckle noise in the data, all data sets are pre-processed with anisotropic diffusion, as presented in [5]. Manual initialization of OF tracking was performed at end-diastole (ED) via tracing of the endocardial and epicardial surfaces. The OF algorithm was applied to derive motion from ED to end-systole (ES). Myocardium motion fields were derived with four different schemes. Finally, cardiac dynamic measurements (displacements and their derivatives) were computed as illustrated in the computational flowchart provided in Figure 1.

\subsection{Manual Segmentation}

An expert performed manual tracing of the ED endocardial and epicardial surfaces, on rotating B-scan views (long-axis views rotated around the base-to-apex axis of the ventricle) and C-scan views (short-axis views at different depths). These borders were used to initialize the $\mathrm{OF}$ algorithm and generate a myocardial mask to guide downstream processing (as shown in Figure 1). 


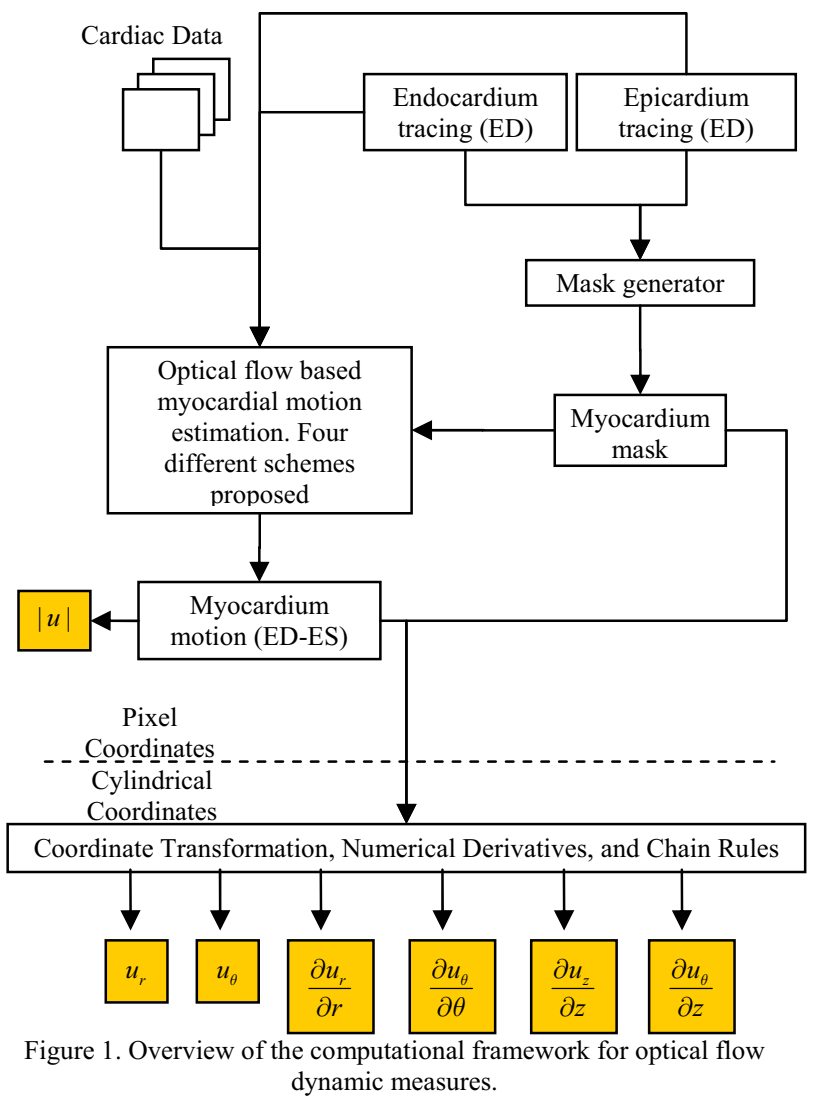

\subsection{Correlation-based optical flow}

OF tracking refers to the computation of the displacement field of objects in an image, based on the assumption that the intensity of the object remains constant. There are two global families of $\mathrm{OF}$ computational techniques: (1) differential techniques [6-8] that compute velocity from spatio-temporal derivatives of pixel intensities and (2) region-based matching techniques $[9,10]$, which compute the OF via identification of local displacements corresponding to optimal correlation of two consecutive image frames. Compared to differential OF approaches, region-based methods using correlation measures are less sensitive to noise and fast motion [11] but assume that displacements in a small neighborhood are similar. In the context of RT3D ultrasound, this later approach appeared to be more appropriate and was selected for this study. Thus, correlation-based OF was applied to estimate the displacement of selected voxels between two consecutive ultrasound volumes in the cardiac cycle. The search window $\Omega$ was set to size $(7 \times 7 \times 7)$ and centered at every $(5 \times 5 \times 5)$ voxel.

\subsection{Myocardial motion field computation}

In this paper, we investigated four different schemes to derive a myocardial motion field:
Scheme 1: Boundary tracking with RBF interpolation [12]. As we proposed in [4], ED endocardial and epicardial boundary points were used to initialize the OF tracking of the myocardial surfaces at ES. The inside myocardial motion field was then estimated by radial basis function interpolation from the myocardial surfaces.

Scheme 2: Direct tracking within a myocardial mask. A straightforward alternative to scheme 1 is to track with OF every voxel within a myocardial mask defined by the myocardial surfaces, instead of interpolating the $\mathrm{OF}$ tracking result of these surfaces.

Scheme 3: Full-field OF estimation. A more global approach consists of estimating the motion field for all the voxels in the input volumes using OF. The myocardial motion field is then extracted by masking the motion field of the voxels belonging to the myocardium.

Scheme 4: Full-field OF estimation with smoothing. The RBF interpolation of scheme 1 provided a $2^{\text {nd }}$-order continuity which is not used in direct OF estimation. We tested an alternative to Scheme 3 by adding smoothing via cubic spline regularization on the full field $\mathrm{OF}$ computation.

\subsection{Coordinates Systems}

Three coordinate systems are involved in the computational framework depicted in Figure 2: pixel coordinates $(i, j, k)$, Cartesian coordinates $(x, y, z)$, and cylindrical coordinates $(r, \theta, z)$. The $\mathrm{OF}$ estimation was performed in pixel coordinates. For computation of dynamic information, displacements in pixel coordinates were converted into Cartesian coordinates and centered inside the ventricular cavity with the z-axis aligned with the long axis of the left ventricle (via rigid transformation). The ventricular axis was defined as the axis connecting the center of the mitral orifice and the endocardial apex. This axis can be easily defined throughout the cardiac cycle [13]. Based on the Cartesian coordinate system, a corresponding cylindrical coordinate system was established with the $r-\theta$ plane aligned with the $\mathrm{x}-\mathrm{y}$ plane and the $\mathrm{x}$-axis taken as the reference for $\theta$.

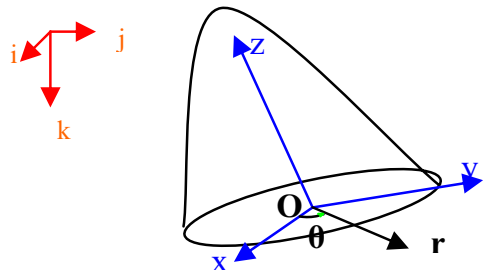

Figure 2. Coordinate systems for data acquisition and computation.

\subsection{Dynamic cardiac measurements}

In addition to the displacement $\left(u_{x}, u_{y}, u_{z}\right)$ in Cartesian coordinates, we can compute the following dynamic measurements: 
- Flow magnitude $|u|(\mathrm{mm})$

- Radial displacement $u_{r}(\mathrm{~mm})$

- Circumferential displacement $u_{\theta}(\mathrm{mm})$

- Thickening $\partial u_{r} / \partial r$

- Circumferential stretch $\partial u_{\theta} / \partial \theta$

- Longitudinal stretch $\partial u_{z} / \partial z$

- Twist $\partial u_{\theta} / \partial z$

Gradient values were computed directly in pixel coordinates and converted into the cylindrical coordinate system via chain rules. Derivatives in pixel coordinates were approximated by central difference operators.

In clinical applications, a 16-segment model of the left ventricular myocardium is used according to a standard partitioning defined by the American Society of Echocardiography [14]. According to this partitioning, we computed average values of the proposed dynamic cardiac metrics within each segment.

In a recent cardiac biomechanics study [15], thickening was found to be the most sensitive measurement to distinguish ischemic from non-ischemic myocardial regions. Although the images in that study were 3D MRI images, the coordinate setting was similar to ours. The "thickening" defined in their study corresponded to the segmental averaged results on the "thickening" measurements based on displacement derivatives as defined here.

We therefore focused our efforts on "thickness" related metrics for the quantitative evaluation of our myocardial motion analysis method: comparing optical flow magnitude, radial displacement, and thickening.

\subsection{Data}

The proposed tracking approach was tested on one data set acquired with a Philips SONOS 7500 3D ultrasound machine. This data set consisted of a transthoracic clinical examination of a heart-transplanted patient. Spatial resolution of the analyzed data was $0.8 \mathrm{~mm}^{3}$ and 16 frames were acquired for one cardiac cycle. Due to the hearttransplant, the patient had reduced cardiac function. Three cross-sectional views at ED are shown in Figure 3. Data acquisition was initialized at ED and synchronized with the ECG. Septal wall motion abnormalities are commonly found in patients after cardiac surgery.

Due to field of view limitations, the apical epicardial surface was not visible in the ultrasound volume. Thus only the basal and middle parts of left ventricle were used for dynamic analysis. However, myocardial shape in this region can be well approximated by a cylinder, which can reduce geometric errors in radial displacement estimation. A global motion of 2-pixel translation at the apex on the endocardial surface and a $2^{\circ}$ rotation of the ventricular axis were observed during systole.

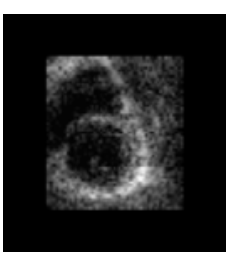

(a)

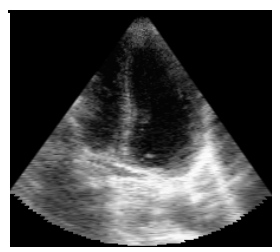

(b)

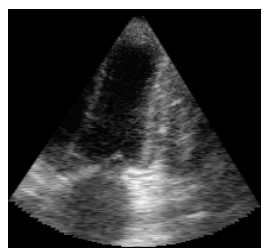

(c)
Figure 3. Original data: (a) short-axis (b) elevation, (c) azimuth views.

\section{RESULTS AND DISCUSSION}

The OF tracking results from the four different schemes were compared head to head in the following figures. The results showed several facts:

- The radial displacement fields derived from the four different schemes were similar except for fine details within the myocardium. This is expected since all the methods depended on the OF tracking results. For the radial thickening, Scheme 1, 3, and 4 provided similar results as well whereas Scheme 2 produced flawed results due to the derivative calculation across the boundary.

- The thickening values of the normal part of the wall from Schemes 1, 3, and 4 (around 0.1 0.25) were close to the normal values reported in [16] (0.1 0.4) and [17] (20$40 \%)$.

- The segmental averaged thickening result showed that anterior and lateral segments had normal motion whereas the septal segment had outward motion and negative thickening values; the posterior and anteroseptal segments had reduced motion; the inferior segments had very small motion or thickening.

- All schemes required the same amount of manual initialization, i.e. endo- and epicardial tracing at ED. In terms of accuracy, Scheme 3 was more accurate in displacement estimation; however, Schemes 1 and 4 were more robust in estimating thickening, benefited from their intrinsic smoothness constraints. Scheme 1 was the most computationally efficient, using an optimized RBF interpolation implementation.

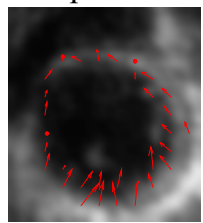

(a)

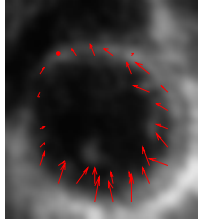

(b)

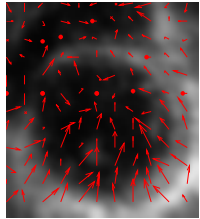

(c)

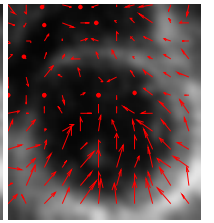

(d)
Figure 4. Optical flow field computed from the four different schemes: (a)-(d): Schemes 1-4.

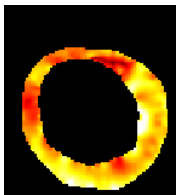

(a)

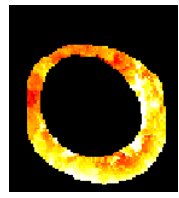

(b)

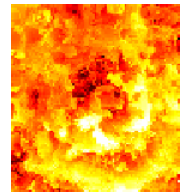

(c)

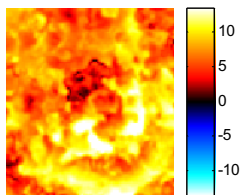

(d)
Figure 5. Flow magnitude (in voxels) derived from the four different schemes: (a)-(d): Schemes 1-4. 


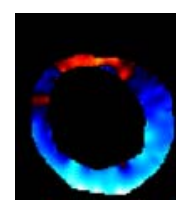

(a)

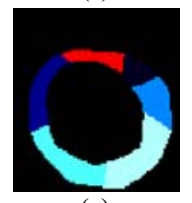

(e)

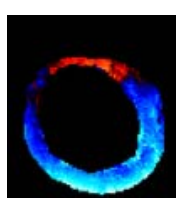

(b)

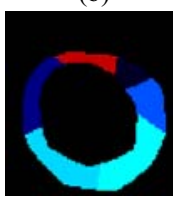

(f)

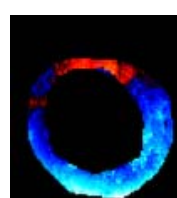

(c)

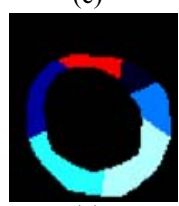

(g)

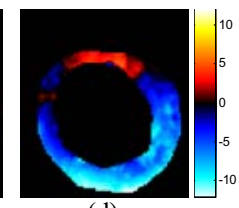

(d)

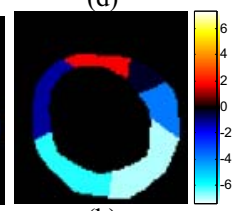

(h)
Figure 6. Radial displacement (in voxel) from the four different schemes: (a)-(d): voxel distribution from Schemes 1-4; (e)-(h) segmental average from Schemes 1-4.

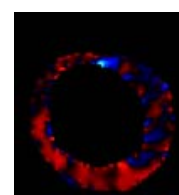

(a)

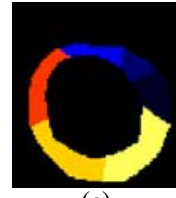

(e)

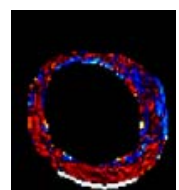

(b)

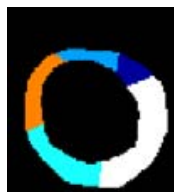

(f)

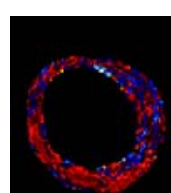

(c)

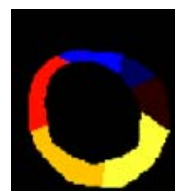

(g)

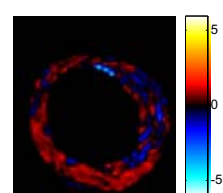

(d)

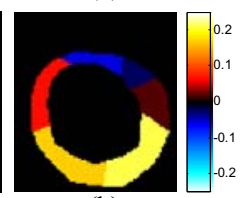

(h)
Figure 7. Thickening (in 100\%) from the four different schemes: (a)-(d): voxel distribution of Schemes 1-4; (e)-(h) segmental average of Scheme $1-4$.

\section{CONCLUSION}

A 4D, correlation-based, optical flow algorithm was applied to RT3D ultrasound data for tracking endocardial and epicardial surfaces. Dense myocardium motion fields were recovered via four different schemes and dynamic cardiac measurements, such as radial displacement and thickening, were computed. Preliminary results showed that the scheme with the lowest computational complexity, which consisted of boundary tracking with RBF interpolation, yielded similar results to full-field OF with or without smoothing. Myocardial thickening values computed from Schemes 1, 3, and 4 for the normal part of the LV wall also matched normal values reported in the literatures. In the future, more extensive evaluation will be performed.

\section{ACKNOWLEDGEMENTS}

This work was funded by National Science Foundation grant BES-02-01617, American Heart Association \#0151250T, Philips Medical Systems, New York State NYSTAR/CAT Technology Program, and the Louis Morin Fellowship program. The authors also would like to thank Dr. Pulerwitz (Department of Medicine, Columbia University).

\section{REFERENCES}

1] O. T. V. Ramm and S. W. Smith, "Real time volumetric ultrasound imaging system," Journal of Digital Imaging, vol. 3, pp. 261-266, 1990.

[2] X. Papademetris, A. J. Sinusas, D. P. Dione, et al., "Estimation of 3D left ventricular deformation from echocardiography," Medical Image Analysis, vol. 8, pp. 285-294, 2004.

[3] Q. Duan, E. D. Angelini, S. L. Herz, et al., "Evaluation of Optical Flow Algorithms for Tracking Endocardial Surfaces on ThreeDimensional Ultrasound Data," presented at SPIE International Symposium, Medical Imaging 2005, San Diego, CA, USA, 2005.

[4] Q. Duan, E. Angelini, S. L. Herz, et al., "Dynamic Cardiac Information From Optical Flow Using Four Dimensional Ultrasound," presented at 27th Annual International Conference IEEE Engineering in Medicine and Biology Society (EMBS), Shanghai, China, 2005

[5] Q. Duan, E. D. Angelini, and A. Laine, "Assessment of visual quality and spatial accuracy of fast anisotropic diffusion and scan conversion algorithms for real-time three-dimensional spherical ultrasound," presented at SPIE International Symposium Medical Imaging, San Diego, CA, USA, 2004.

[6] B. D. Lucas and T. Kanade, "An iterative image registration technique with an application to stereo vision," presented at International Joint Conference on Artificial Intelligence (IJCAI), 1981.

[7] B. K. P. Horn and B. G. Schunck, "Determing optical flow," Artificial Intelligence, vol. 17, pp. 185-203, 1981.

[8] H. Nagel, "Displacement vectors derived from second-order intensity variations in image sequences," Computer Vision Graphics Image Processing, vol. 21, pp. 85-117, 1983.

[9] P. Anandan, "A computational framework and an algorithm for the measurement of visual motion," International journal of Computer Vision, vol. 2, pp. 283-310, 1989.

[10] A. Singh, "An estimation-theoretic framework for image-flow computation," presented at International Conference on Computer Vision, 1990

[11] J. L. Barron, D. Fleet, and S. Beauchemin, "Performance of optical flow techniques," Int. Journal of Computer Vision, vol. 12, pp. 4377, 1994.

[12] B. J. C. Baxter, "The Interpolation Theory of Radial Basis Functions," Cambridge University, 1992, pp. 1-142.

[13] S. Herz, T. Pulerwitz, K. Hirata, et al., "Novel Technique for Quantitative Wall Motion Analysis Using Real-Time ThreeDimensional Echocardiography," presented at Proceedings of the 15th Annual Scientific Sessions of the American Society of Echocardiography, 2004.

[14] N. B. Schiller, P. M. Shah, M. Crawford, et al., "Recommendations for quantitation of the left ventricle by two-dimensional echocardiography. American Society of Echocardiography Committee on Standards, Subcommittee on Quantitation of TwoDimensional Echocardiograms," J Am Soc Echocardiogr, vol. 2, pp. 358-367, 1989.

[15] H. Azhari, S. Sideman, J. L. Weiss, et al., "Three-dimensional mapping of acute ischemic regions using MRI: wall thichening versus motion analysis," American Journal of Physiology, vol. 259, pp. H1492-1503, 1990.

[16] L. K. Waldman, Y. C. Fung, and J. W. Covell, "Transmural myocardial deformation in the canine left ventricle. Normal in vivo three-dimensional finite strains," Circ Res, vol. 57, pp. 152-163, 1985.

[17] M. Nieminen, A. F. Parisi, J. E. O'Boyle, et al., "Serial evaluation of myocardial thickening and thinning in acute experimental infarction: identification and quantification using two-dimensinoal echocardiography," Circulation, vol. 66, pp. 174-180, 1982. 